\title{
Is Clinical Anxiety a Risk or a Protective Factor for Executive Functioning in Youth with ADHD? A Meta-regression Analysis
}

\author{
Marija Maric ${ }^{1} \cdot$ Anika Bexkens $^{2,3} \cdot$ Susan M. Bögels ${ }^{4}$
}

Published online: 26 February 2018

(c) The Author(s) 2018. This article is an open access publication

\begin{abstract}
Comorbidity rates between ADHD and anxiety disorders (AD) are high, but little is known about the nature of this cooccurrence. A dominant idea is that AD may intensify some (i.e., attention and working memory) and attenuate other (i.e., inhibition) ADHD symptoms. Results are mixed, potentially because of between-study differences. To investigate this further we performed a meta-regression analysis on 11 studies ( $n$ 'ADHD-only' $=695 ; n$ 'ADHD + AD' $=608$ ), containing 35 effect sizes on attention, inhibition and working memory. Main results were: (1) no evidence of a negative effect of $\mathrm{AD}$ on attention and working memory; (2) better response inhibition in children with ADHD with AD than those with only ADHD (medium ES $g=-.40$ ); (3) medication moderated this association: the effect seemed limited to studies that included medication-naïve participants; (4) the difference between the two groups increased with age for attention and with proportion of boys for working memory ability. There was no effect of comorbid disruptive behavior disorder. In conclusion, AD seems to be a protective factor for inhibition problems as assessed with laboratory tasks in ADHD, especially in children who are medication naïve. Further, AD may have a protective function for attention in older children, and for working memory in boys with ADHD. It is therefore important to screen for $\mathrm{AD}$ when diagnosing $\mathrm{ADHD}$, and to educate those with comorbid $\mathrm{AD}$ about the possible positive function of feeling anxious. Potential negative effects of ADHD medication on inhibition in children with comorbid AD should be considered.
\end{abstract}

Keywords Attention-deficit/hyperactivity disorder (ADHD) $\cdot$ Anxiety disorder $\cdot$ Attention $\cdot$ Response inhibition $\cdot$ Working memory $\cdot$ Meta-analysis

Marija Maric and Anika Bexkens contributed equally to this article.

Marija Maric

m.maric@uva.nl

1 Department of Developmental Psychology, University of Amsterdam, Nieuwe Achtergracht 129B, 1018 WT Amsterdam, The Netherlands

2 Department of Developmental Psychology, Leiden University, Leiden, The Netherlands

3 Department of Child and Adolescent Psychiatry, Center for Psychiatry, GGZ Delfland, Delft, The Netherlands

4 Research Institute of Child Development and Education, University of Amsterdam, Amsterdam, The Netherlands

\section{Introduction}

The comorbidity of attention-deficit/hyperactivity disorder (ADHD) and anxiety disorders (AD) is substantial with average comorbidity rates between 25 and $50 \%$ found in both epidemiological as well as in clinical samples (e.g., Angold et al. 1999; Jarrett and Ollendick 2008; Mancini et al. 1999; MTA group 1999). Moreover, the comorbidity of the two conditions occurs across all life stages (e.g., Kessler et al. 2006; Cumyn et al. 2009; Posner et al. 2007) and in different nationalities (e.g., Park et al. 2011; Smalley et al. 2007; Sobanski et al. 2007).

Although the comorbidity rates of ADHDs and ADs are well-documented, little is known about the etiology of this condition, nor its treatment response. Theories of ADHD suggest that deficits in executive functions (EFs) play an important role in explaining the common ADHD symptoms, and problems children with ADHD encounter in daily lives (Barkley 2012; Nigg 2006). Though the 
definition of executive functions is still evolving, most authors do agree that EFs involve meta-cognitive or meta-attentional processes needed to regulate own behavior, thought or emotion, and pursue daily and broader life goals. As for ADHD, the idea is that, e.g., behavioral manifestations of the disorder such as impulsivity and hyperactivity are largely driven by difficulties in EFs such as attentional control (i.e., "paying attention and staying on task"; Nigg 2006), response inhibition (i.e., "the ability to stop a previously prepared response"; Nigg 2006) and working memory (i.e., "maintaining the amount of information online and to activate some information over other"; Nigg 2006). Indeed, deficits in several EFs have been identified in individuals with ADHD, and, at this point in time, research continues to affirm cognitive deficits involved in the etiology of ADHD (Burgess et al. 2010; Crosbie et al. 2013; Kofler et al. 2010; Sarver et al. 2015; Tillman et al. 2011). Perhaps predictably, emerging empirical studies concerned with investigations of the cooccurrence of ADHD and ADs have been mainly directed at the assessment of EFs in these comorbid populations.

So far, three independent literature reviews concerned with the impact of ADs on executive functioning in ADHD have been conducted including articles from 1989 to 2009 (Pliszka et al. 1999; Schatz and Rostain 2006; Tannock 2009). Consequently, these authors concluded that the presence of anxiety in individuals with ADHD may both intensify and attenuate common ADHD difficulties such as EF deficiencies. More specifically, anxiety may negatively interfere with cognitive functioning, making attentional, working memory, and other cognitive deficits worse. On the other hand, anxiety can reduce impulsivity and response inhibition deficits seen in ADHD. Since then, this has become one of the main research domains in the area of comorbid ADHD and anxiety, and several additional empirical studies have been conducted investigating this topic. The results have been mixed, potentially because of the between-studies differences. For example, while some studies allowed for other comorbidities (i.e., Oppositional Defiant Disorder [ODD] and Conduct Disorder [CD]) to be present in the comorbid ADHD and ADs samples (e.g., Vloet et al. 2010), others included purer comorbid ADHD and ADs samples (e.g., Jarrett et al. 2016). To investigate this idea that anxiety is both a risk (i.e., for attention and working memory problems) and a protective (i.e., for response inhibition problems) factor for executive functioning problems in ADHD, our first aim was to perform a meta-analysis on studies that have assessed the impact of comorbid $\mathrm{AD}$ on attentional, response inhibition, and working memory capacities in youths with ADHD. This proposition is thus mainly driven by theory, clinical experience and conclusions from previous review studies
(Pliszka et al. 1999; Schatz and Rostain 2006; Tannock 2009).

The second aim of this study is to identify variables associated with several participants' characteristics that may moderate the relationship between $\mathrm{AD}$ in children with ADHD and executive functioning problems. Given the contradictory findings related to the effects of ADHD medication in youth with comorbid ADHD and ADs, medication status was studied as a potential moderator. While some studies found a positive effect of medication on ADHD behavioral symptom outcomes in children with ADHD and comorbid anxiety (MTA group 1999), other studies found no effect of medication (Diamond et al. 1999), or even adverse medication effects on anxiety symptoms and cognitive functioning (Bedard and Tannock 2008; Pliszka 1989; Tannock et al. 1995). Next to medication status, ODD/CD was investigated as a moderator given that: (a) approximately $50 \%$ of children and adolescents with ADHD also have a comorbid CD/ODD (e.g., MTA group 1999); (b) studies have revealed broad ranges of comorbidity for $\mathrm{AD}$ and ODD/CD (Ollendick et al. 2008); and (c) there is an emerging evidence for EF deficiencies in children with CD/ODD (e.g., Schoemaker et al. 2013). Finally, as there is an age-related increase in the prevalence of comorbid anxiety in individuals with ADHD (e.g., Biederman et al. 1994), females with ADHD report higher levels of comorbid anxiety (Biederman et al. 1994; Rucklidge and Tannock 2001), and no differences in executive functioning impairment were found between adolescent boys and girls with ADHD (Seidman et al. 2005), we aimed to investigate the moderating roles of age and gender. Given the diverse findings from previous studies and the novelty of this topic, we did not formulate specific hypotheses with regard to above-discussed potential moderators; the moderation analyses were exploratory in nature.

The current study updates previous literature reviews (Pliszka et al. 1999; Schatz and Rostain 2006; Tannock 2009) with 6 new studies. More important, this is the first meta-analytic review concerned with the role of anxiety in ADHD, and the first attempt to investigate a range of moderating effects related to participants' characteristics on EF functioning in individuals with ADHD (and comorbid AD). Investigation of moderators is crucial in this population given the high heterogeneity of the ADHD disorder (Nigg 2006), and thus diverse information included within single studies, but also important given the large between-studies differences.

\section{Methods}

\section{Literature Search}

The search for studies comprised electronic databases (PsycINFO, PubMed, Web of Science, Google Scholar) using 
the following keywords: attention-deficit hyperactivity disorder, attention-deficit disorder, hyperactivity, ADHD, ADD, executive function*, attention* (response), inhibit*, working memory cross-referenced with anx* and comorbid*. Next, reference lists of reviews and book chapters were reviewed, and Google pages were searched for unpublished theses and dissertations. Furthermore, experts in the field were presented with the list of studies produced by the searches outlined above and were asked to add any further (un)completed studies that they were aware of. The search was performed by the research assistant under supervision of MM. The flow chart of the selection process is shown in Fig. 1, in the corresponding PRISMA chart (Moher et al. 2009).

\section{In- and Exclusion Criteria}

The review was restricted to empirical studies reporting on individuals diagnosed with attention-deficit/hyperactivity disorder (ADHD) according to DSM (-5, or -IV-TR) criteria (American Psychiatric Association 2000; 2013) or previous editions. Articles were included when meeting the following criteria: (a) comparison of ADHD clinical group without comorbid AD or (sub)clinical levels of symptoms, with an
ADHD clinical group with comorbid AD or (sub)clinical levels of symptoms; (b) the two groups were compared on at least one executive function of interest, i.e., attention, working memory, response inhibition; (c) group means as well as standard deviations on outcome measures were reported in the articles (or were retrieved through correspondence with the authors). While a DSM ADHD diagnosis was a strict inclusion criterium of the meta-analysis, articles relying on either DSM anxiety diagnosis or standardized anxiety questionnaires in order to select their comorbid group were included in the meta-analysis. Further, only etiology studies on this topic were included, and not treatment outcomes studies (utilizing experimental manipulation on one of the outcome or moderating variables).

Studies were excluded if executive functions were proposed to be assessed by parts of the DSM diagnostic interview (e.g., items assessing ADHD—inattention symptoms) or via self or parent or teacher report questionnaires (e.g., Sørensen et al. 2011) as we assumed that these types of assessment methods may produce ratings of ADHD DSM symptom presentations and behavioral manifestations of executive functioning instead of EFs as underlying neurocognitive processes. Several studies we traced did not
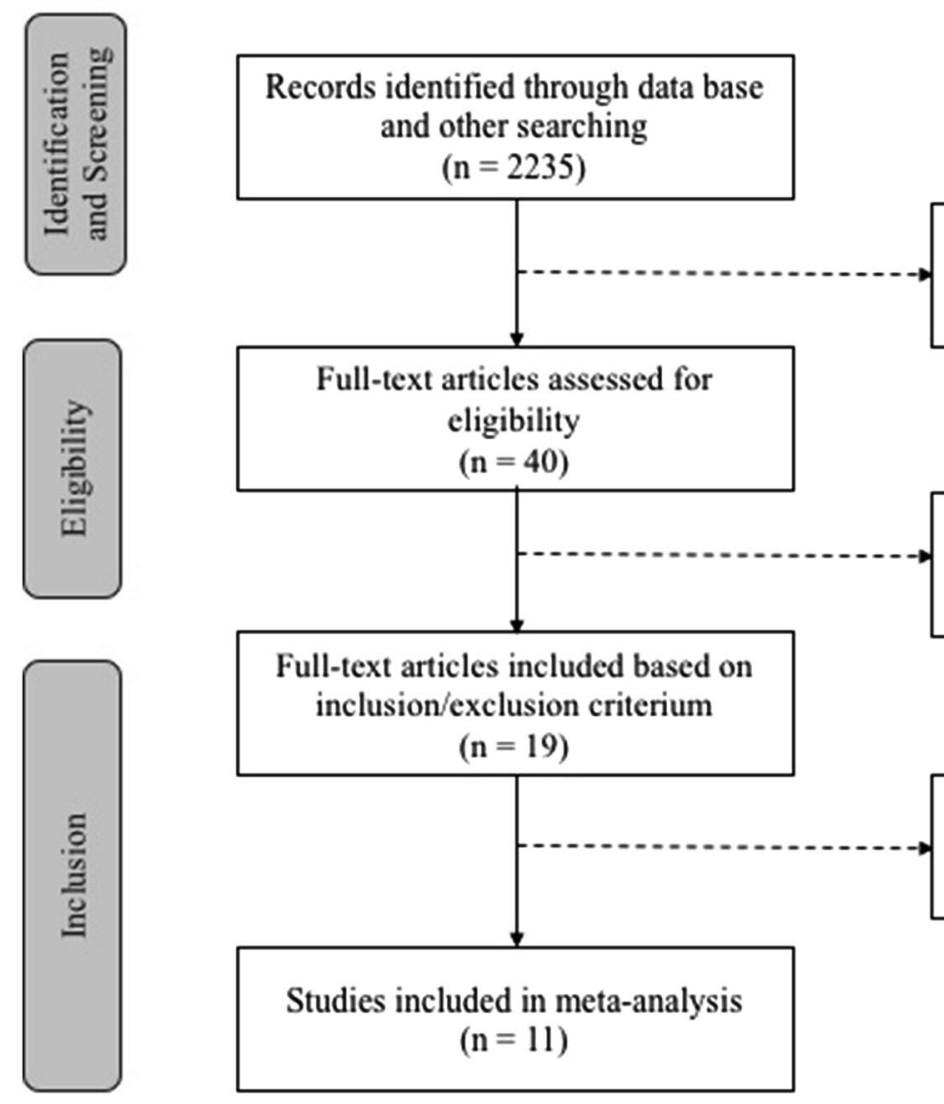

Records excluded based on title and/or abstract $(\mathrm{n}=2195)$

Full-text articles excluded based on inclusion/exclusion criterium $(\mathrm{n}=21)$

Full-text articles excluded based on unavailability of relevant parameters $(\mathrm{n}=8)$

Fig. 1 PRISMA flowchart of screened and included studies 
include direct comparison of ADHD without anxiety and ADHD with anxiety groups on EFs, but were, for example, concerned with associations between EFs and anxiety symptoms in ADHD group-only or used CBCL-Internalizing scale (containing anxiety and depression items) to define the comorbid group. We tried to retrieve the information on the right parameters from these authors, and in case that this information was available, it was included in the meta-analysis.

\section{Selection and Coding of the Studies}

MM reviewed the titles and abstracts of the studies retrieved during literature search and selected 40 studies. AB and SMB coded independently these 40 articles according to: inclusion of two groups in the article, a group with ADHD diagnosis and a group with comorbid ADHD and AD/ symptoms, and investigation of EFs across the two groups. Inter-rater reliability of this first step was .95. In the second step, AB and SMB coded whether articles compared the two groups on EFs attention, working memory and response inhibition; the inter-rater reliabilities were .97, .93, and .93, respectively. Discrepancies were resolved through discussions. Table 1 provides a complete list of included studies $(n=11)$, moderating variables and effect size estimates.

\section{Assessment of EFs}

$\mathrm{MM}$ and $\mathrm{AB}$ reviewed assessment tools used in different articles to ensure that only the information which is in line with goals of this meta-analysis, that is, examining the impact of $\mathrm{AD}$ on EFs as underlying cognitive processes in youth with ADHD, is included in our study. Assessment tools proposed to measure attention were directed at assessing the effect of distracting stimuli on primary task performance of an individual. Instruments included were different versions of the Continuous Performance Test $(n=4)$, D2 test $(n=1)$, TOVA $(n=1)$, Bell test $(n=1)$, alertness task $(n=1)$, sustained attention task $(n=1)$, go/no-go RTV $(n=1)$, and divided attention task $(n=1)$. Assessment tools proposed to measure response inhibition required withholding a prepotent/automatic or ongoing response in mind. Instruments included were stoptask $(n=2)$, Continuous Performance Test $(n=2)$, go/no-go $(n=1)$, Stroop $(n=1)$. Assessment tools proposed to measure working memory were directed at participants having to keep information in mind during a delay, and manipulate the information. Instruments included were WISC-III Digit span backward $(n=2)$, WISC-R Digit span backward/forward $(n=1)$, F-Digit backward $(n=1)$, WRAML $(n=1)$, Chipasat $2.0(n=1)$, Chipasat $2.8(n=1)$, CANTAB $(n=1)$, Corsi block tap test $(n=2)$. An overview of the assessment instruments as well as related parameters is presented in Table 1.

\section{Anxiety Severity}

In all but one study (Rodríguez et al. 2014) anxiety was determined based on formal DSM (-III, -IV, -5) diagnosis using the following clinical interviews: ADIS-C/P (Jarrett et al. 2016; Manassis et al. 2007), PICS-IV (Korenblum et al. 2007), DICA-R-P (Manassis et al. 2000), DISC (Newcorn et al. 2001), a structured DSM-III-R interview (Pliszka 1992), K-SADS-PL (Trani et al. 2011; Vance et al. 2013; Yurtbasi et al. 2015), and KDIPS (Vloet et al. 2010). In Rodríguez et al. (2014) study, inclusion in the ADHD + AD group was based on the trait score on STAI-C questionnaire and all cases had a score higher than $90 \%$.

\section{Meta-regression Procedure}

In meta-regression, it can be tested whether the overall effect size in a set significantly deviates from zero. Moreover, it is possible to test whether study or participant characteristics of the selected studies moderate the size of effects. We used a random-effects meta-regression model, which accounts for between-study variation in effect sizes using R package: metafor (Viechtbauer 2010). The analysis provides an estimate of the overall effect, tests of the effects of moderators and a test of between-study variation $(Q E$ statistic). The standardized difference between two means, Hedges' g, was calculated for each comparison of ADHDonly versus $\mathrm{ADHD}+\mathrm{AD}$ on $\mathrm{EF}$ tasks within the selected studies in such a way that negative effect sizes represent larger executive function problems in ADHD-only, whereas positive effect sizes represent larger executive function problems in ADHD + AD. Studies were weighted by sample size. Several studies included more than one task to measure executive function, or used tasks that resulted in more than one parameter indexing executive function ability. To correct for dependency between effect sizes from the same study, multilevel meta-regression was used estimating variance within and between studies. We ran random-effects meta-regression without moderators for each EF separately. Besides reporting on the confidence intervals (CIs) which quantify the precision of an estimated effect, in the results we also report prediction intervals (PIs) which present the expected range of true study effects in similar, new studies (IntHout et al. 2016). It can be particularly informative to inspect PIs when there is high heterogeneity between studies included in a meta-analysis (IntHout et al. 2016; Partlett and Riley 2017). Subsequently, we ran separate analyses (QM tests; Viechtbauer 2010) for each EF including one moderator at a time. As these analyses were exploratory, we consider the results to be hypothesis forming, instead of hypothesis testing. We used Cohen's guidelines (1992) in interpreting the size of overall effect sizes. In line with 


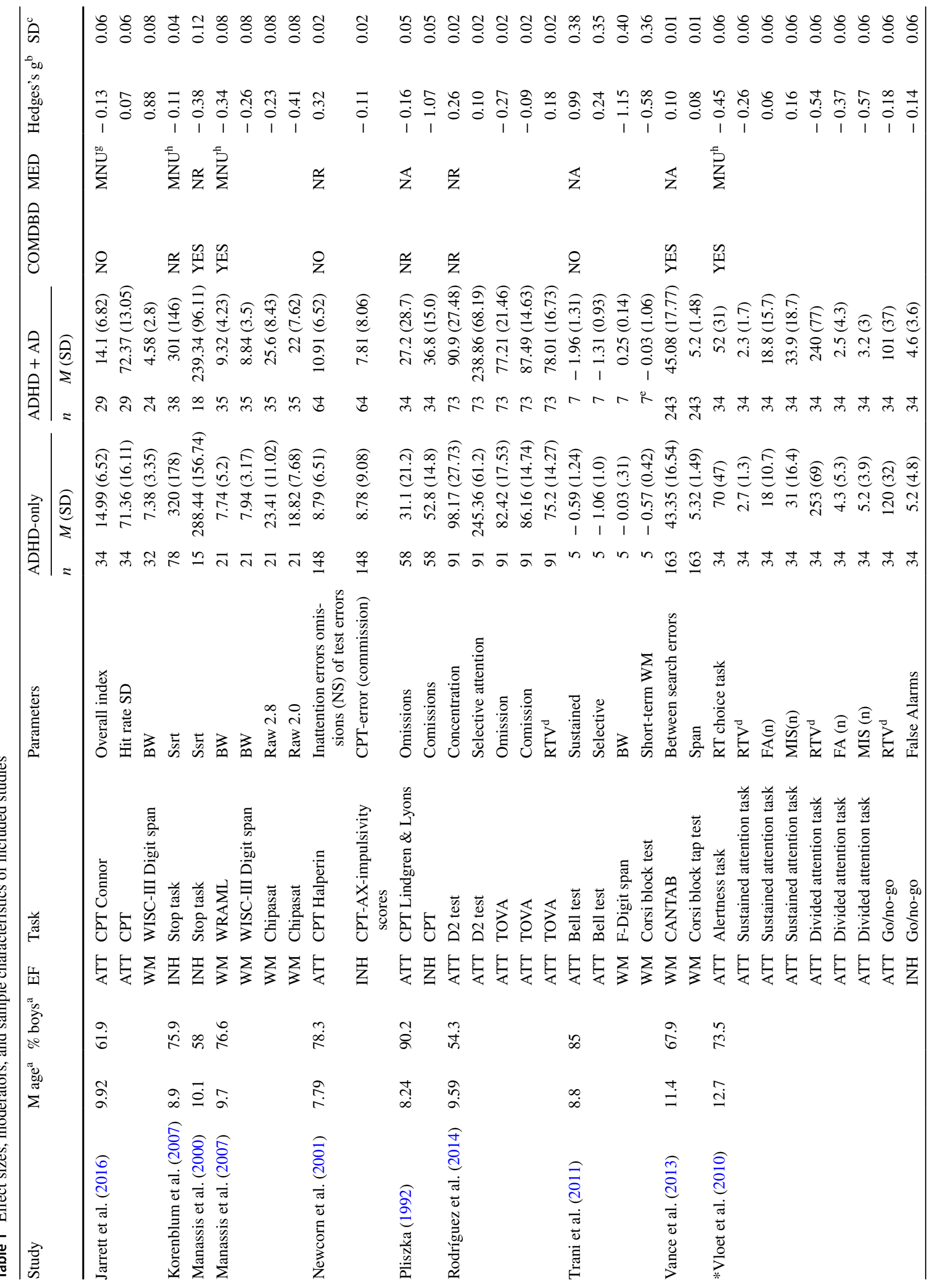




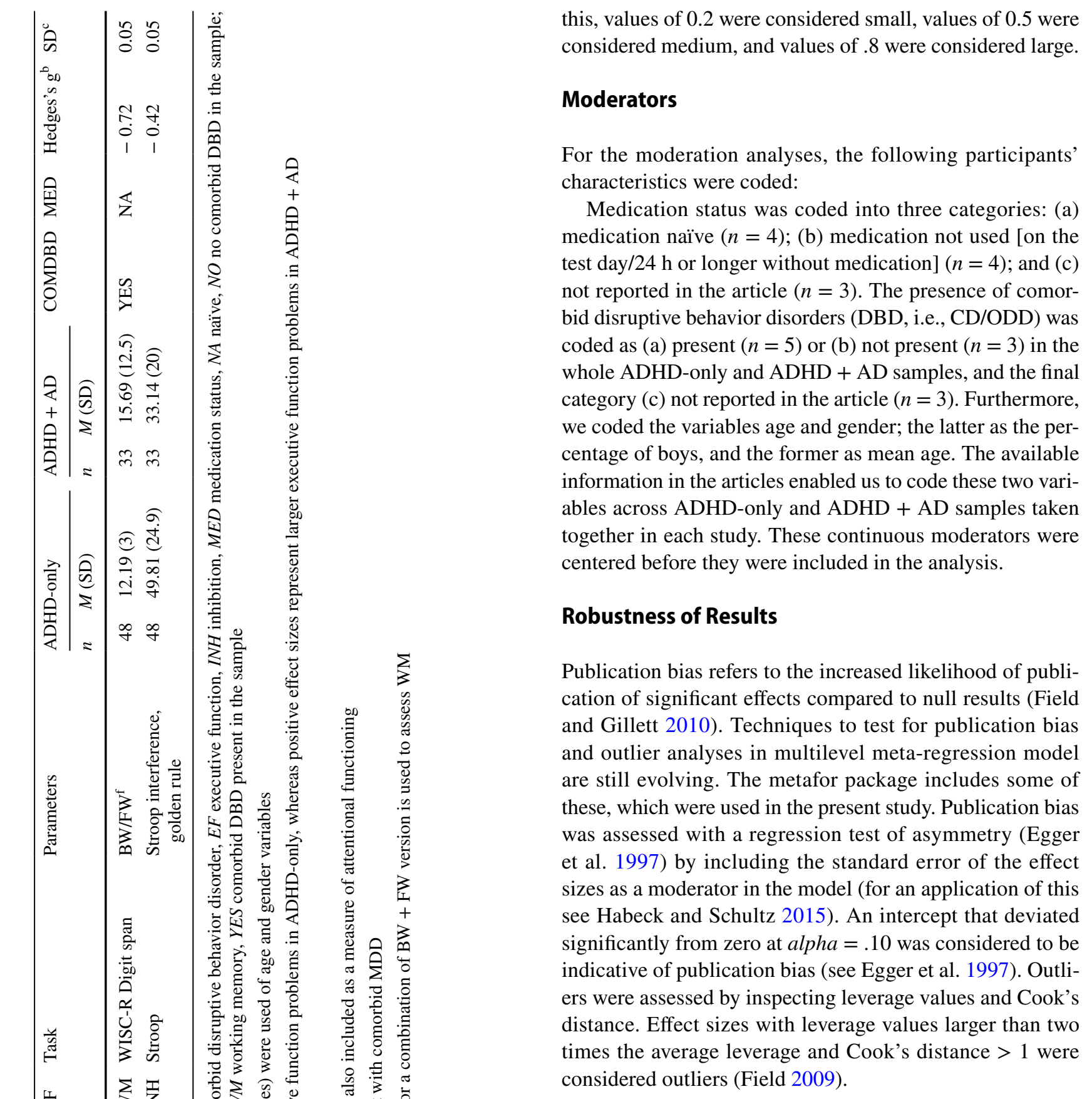

\section{Results}

The literature search and selection procedure described above resulted in 11 studies that were included in the metaregression, and a total of 35 effect sizes that compared ADHD-only and ADHD + AD groups on attention $(n=19)$, inhibition $(n=6)$ and working memory $(n=10)$ parameters. Negative effect sizes represent larger executive function problems in ADHD-only, whereas positive effect sizes represent larger executive function problems in ADHD + AD. Effect sizes varied between large negative $(-1.15)$ and large 
positive (0.99; cf Table 1). See Fig. 2a-c for funnel plots representing variation in effect sizes within each subset of executive functioning.

To test our first proposition that comorbid anxiety has a differential effect on different executive functions, we performed a random-effects meta-regression model with executive function type as moderator (i.e., "executive function type' as independent variable, "effect size" as dependent variable). The analysis showed that overall $\mathrm{EF}$ was not a significant moderator of effect size (cf. Fig. 3a-c for forest plot). The omnibus test is not significant $(\mathrm{QM}=4.24$, $p=.10$, two sided).

However, follow-up tests did reveal that overall effect size in studies of inhibition was significantly larger than overall effect sizes in studies of attention $(p=.04$, two sided) and marginally significantly larger than overall effect sizes on studies of working memory $(p=.09)$. Further analysis showed that only the overall effect size of studies on inhibition deviated significantly from zero. The effect size was medium sized and negative $(b=-.40, p=.004$, CI $-0.67 /-0.13$, two sided), indicating that overall ADHDonly samples presented with larger inhibition deficits than ADHD + AD samples $(n=6)$. There was no significant overall difference between ADHD-only and ADHD + AD on attention or working memory measures. Taken together, these results suggest that anxiety may counteract inhibition problems in ADHD, but not attention or WM problems.

In addition, the analysis showed that there was significant variation between effect sizes, QE $(d f=32)=74.51$, $p<.0001$. Some of this variation may be explained by differences in study characteristics. This was investigated by exploratory moderator analyses per executive function type. We tested age and gender ratio as continuous moderators and Comorbid DBD and Medication as categorical moderators.

\section{Attention}

Overall, effect size of attention parameters did not deviate significantly from zero $(b=-.003, p=.97, \mathrm{CI}-0.21 / 0.20$,
$\mathrm{PI}=-0.47 / 0.47)$. There was significant residual heterogeneity $(\mathrm{QE}(d f=18)=33.33, p=.02)$. Age was a significant moderator of effect size $(p=.002)$, indicating that effect sizes decreased with increasing mean age of the included sample $(b=-.16)$. For this reason age was included as a covariate in the other moderator analyses. With Age as a moderator, there was no significant residual heterogeneity $(\mathrm{QE}(d f=17)=21.19, p=.21)$. None of the remaining moderators (i.e., gender, comorbid DBD or Medication) were significant ( $p$ values $>.86$ ). Taken together these results indicate that the attention difficulties in ADHD-only relative to ADHD + AD increase with age.

\section{Response Inhibition}

Overall, effect size of inhibition parameters significantly deviated from zero $(b=-.41, p=.02, \mathrm{CI}-0.74 /-0.08$, $\mathrm{PI}=-1.15 / 0.33)$. There was significant residual heterogeneity $(\mathrm{QE}(d f=5)=16.97, p=.005)$. Age and gender ratio did not significantly moderate effect size on inhibition tasks ( $p$ values $>.38$ ). Comorbid DBD was not a significant moderator ( $p$ value $>.16$ ). Medication was a significant moderator $(p=<.001)$. After inclusion of medication as a moderator, residual heterogeneity was no longer significant $(\mathrm{QE}(d f=3)=1.64, p=.65)$.

Follow-up tests indicated that overall effect size in studies with medication-naïve samples was significantly smaller (i.e., more negative), than overall effect sizes for studies with samples that were taken off medication for testing $(p<.001)$ or studies were medication status was unknown $(p<.001)$.

Follow-up tests indicated that the overall effect size for studies that included medication-naïve samples deviated significantly from zero $(b=-0.89, p=<.001, \mathrm{CI}$ $-1.21 /-.57, n=2$ ), whereas overall effect size for studies that included samples that were taken off medication for testing $(n=2)$ or studies that did not report medication status $(n=2)$ did not differ significantly from zero $(p$ values $>.27)$.
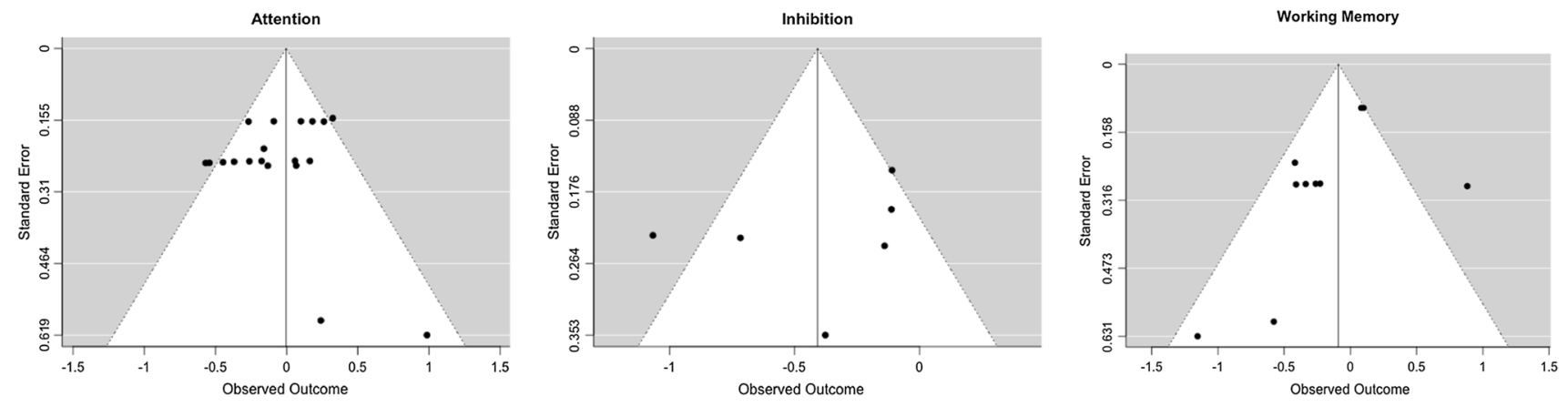

Fig. 2 Funnel plots for effect sizes of the difference between ADHD-only and ADHD + AD on attention, inhibition and working memory tasks 
a

Attention

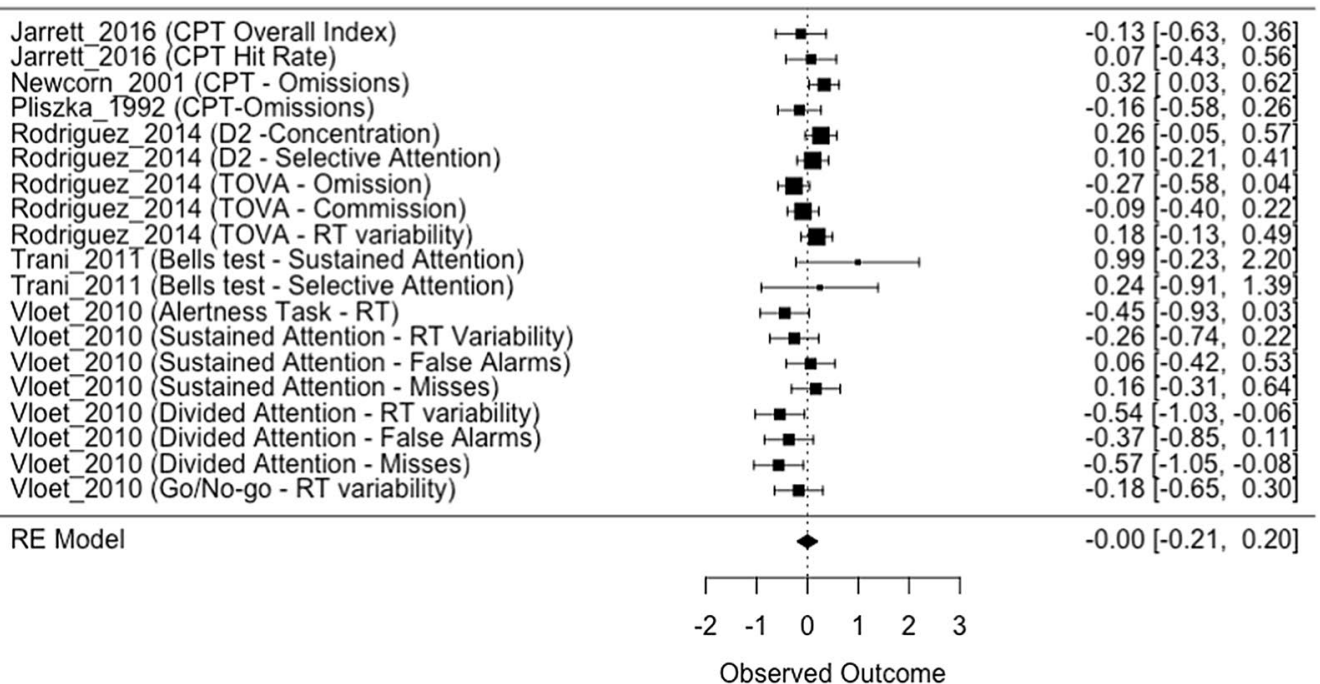

b

Inhibition

\begin{tabular}{ll}
\hline Korenblum_2007 (Stop Signal RT) & $-0.11[-0.50,0.28]$ \\
Manassis_2000 (Stop Signal RT) & $-0.38[-1.07,0.31]$ \\
Newcorn_2001 (CPT-AX - Comissions) & $-0.11[-0.40,0.18]$ \\
Pliszka_1992 (CPT - Comissions) & $-1.07[-1.52,-0.62]$ \\
Vloet_2010 (Go/No-go - False Alarms) & $-0.14[-0.62,0.34]$ \\
Yurtbasi_2015 (Stroop Interference Score) & $-0.72[-1.17,-0.26]$ \\
\hline RE Model & $-2 \quad 0$ \\
& Observed Outcome
\end{tabular}

C

Working Memory

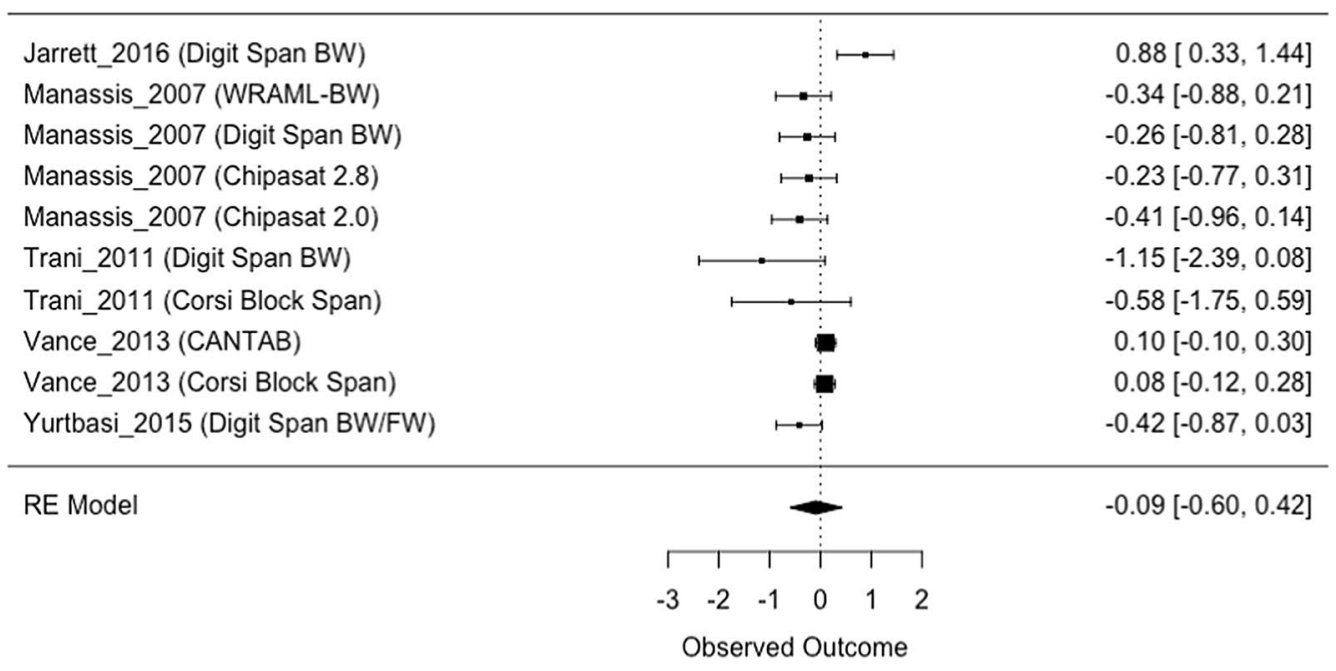


4Fig. 3 Forest plots of effect sizes comparing ADHD-only with $\mathrm{ADHD}+\mathrm{AD}$ samples. Negative effect sizes represent larger executive function problems in ADHD-only, whereas positive effect sizes represent larger executive function problems in ADHD + AD

Taken together these results indicate larger inhibition difficulties in samples with ADHD-only than in samples with ADHD + AD, but only in studies with medicationnaïve participants. In studies that included samples that were not medication naïve there was no overall difference between ADHD-only and ADHD + AD samples on inhibition

\section{Working Memory}

Overall, effect size of working memory parameters did not significantly deviate from zero $(b=-0.09, p=.73$, CI $-0.60 / 0.42, \mathrm{PI}=-1.25 / 1.07)$. There was significant residual heterogeneity $(\mathrm{QE}(d f=9)=24.21, p=.004)$. Age was not a significant moderator of effect size on working memory parameters $(p=.31)$. Gender ratio was a significant moderator of effect size $(b=-.65, p<.001)$, indicating that effect sizes decreased with an increasing proportion of boys in the sample. Inclusion of gender ratio in de model reduced the residual heterogeneity, which was no longer significant $(\mathrm{QE}(d f=8)=6.35, p=.61)$. When further moderator analyses were controlled for gender, comorbid DBD and medication were not significant moderators $(p=.11$ and $p=.13$, two sided, respectively) of effect size on working memory parameters. Taken together, these results indicate that in samples with larger proportion of boys, larger working memory deficiencies are noticed for ADHD-only group than for ADHD + AD group.

\section{Robustness of Results}

We found no evidence of funnel plot asymmetry for the attention, inhibition or working memory subsets of the effect sizes (all $p$ values $>.10$ ). There were no outliers in the attention, inhibition or working memory subsets based on Cook's distance. There were also no studies with an unduly large influence on the results based on leverage values in the inhibition and working memory subsets. In the attention subset, there were two studies with a leverage value higher than two times the average leverage (i.e., Newcorn et al. 2001; Pliszka 1992). We reran the analyses for attention leaving these two effects out. This did not change the pattern of results. The moderating effect of age remained significant $(b=-.17$, $p=.008)$. And the other moderators remained nonsignificant ( $p$ values $>.32$ ). See Fig. 2 for the funnel plots.

\section{Discussion}

Current theories of comorbid ADHD and AD propose that anxiety may both attenuate as well as intensify common ADHD symptoms such as attentional, working memory and inhibition problems (Pliszka et al. 1999; Schatz and Rostain 2006; Tannock 2009). The goal of this meta-analysis was to examine the role of $\mathrm{AD}$ in the executive functions problems of children with ADHD, namely attention, working memory and response inhibition, and test possible moderators in this relationship. Main results were: (1) In line with expectations, children with ADHD plus AD had better response inhibition than those with ADHD alone, with a medium effect size of difference $(g=-.40) ;(2)$ In contrast to expectations, taken over the whole group, we did not find that children with ADHD and AD had more problems in attention and working memory than children with ADHD alone; (3) Of the tested moderators of the association between diagnosis group and executive functioning, ADHD medication, age and gender were significant. Regarding medication, analysis showed that only in studies that included medication-naïve participants, children with ADHD plus AD had better response inhibition than those with ADHD alone. In studies in which medication status was unknown or children were asked to discontinue their medication temporarily, there was no overall difference between children with ADHD plus AD or children with ADHD without AD; (4) moderating analyses indicated further that attentional difficulties in children with ADHD alone seem to increase with age when compared to children with ADHD plus AD, and that working memory difficulties are more present in ADHD alone samples as compared to ADHD plus AD samples when the proportion of the boys in the samples is larger.

The meta-analysis revealed the expected better inhibition in children with ADHD and comorbid AD, which may confirm the proposition that anxiety protects children with ADHD against impulsivity (Pliszka et al. 1999; Schatz and Rostain 2006; Tannock 2009). Being medication naïve seems to be associated with the best inhibition in children with comorbid ADHD and AD. Several reasons can be given for this surprising finding. First, children on medication may be more severe, also on inhibition problems, than children not on medication. This was also demonstrated in a study by Bögels et al. (in preparation), in which of a group of 167 referred children with ADHD who were about to start a mindfulness training, those on medication had more severe ADHD symptoms than those who were medication naïve. At the same time, in our metaanalysis, children in the 'medication naïve' studies were in general somewhat younger than children in other studies included in the meta-analysis, and it seems as if some 
of them were referred for the first time to the clinics. We do not know whether, thus, this argument of differences in severity would hold in the case that these children in medication-naïve studies were older and with treatment histories. Second, it is possible that children who were medication naïve were more borderline cases of ADHD, with primary $\mathrm{AD}$ which may manifest with less specific executive functioning problems such as the ones studied in this meta-analysis. Third, it may not be that these children differ in severity, but the environments they are raised in. Parental own psychopathology and inability to regulate child's behavior, access to alternative help and the extent to which stigma or other social processes interfere with parental decisions to choose for medication may differ between the families (Mueller et al. 2012). At the same time, it could be that anxiety present in these children with ADHD may result in less hyperactive behavior and concentration problems (Schatz and Rostain 2006) and thus cause less constraint in family context enabling the parents to manage their child's behavior more efficiently and without using the medication treatment. As the general idea is that after $24 \mathrm{~h}$ a "wash-out" of ADHD medication takes place (Greenhill et al. 2006), it remains intriguing why we did not find effects of medication in studies with children who did not use medication in the past $24 \mathrm{~h}$ or on test day (but we do find effects of medication in studies with medication-naïve participants). Did these children experience a learning curve while on medication? Or does medication still has effects, even after the "wash-out" period?

We had expected that children with ADHD and comorbid AD would perform worse on attention and working memory measures, as high levels of anxiety and stress could negatively affect attention and working memory (Pliszka et al. 1999; Schatz and Rostain 2006; Tannock 2009). Our null findings therefore require an explanation. First, it might be that high levels of anxiety or stress on a state level do negatively affect attention and working memory, but all but one included study assessed anxiety on a trait level (anxiety disorder diagnosis) and the assessment tools did not necessarily trigger state anxiety. Second, anxiety may have a protective function in children with ADHD, as it enhances attention for threat cues and facilitates sensitivity for the negative consequences of behavior (e.g., fear or punishment). Therefore, it may well be that the null findings result from the combined positive and negative effects of anxiety on attention and working memory. At the same time, a moderating role of age was found for attention; it seems that the difference between ADHD alone and ADHD plus AD groups grows with increasing age with ADHD alone children having more detrimental attentional performance. Therefore, this metaanalysis indicates that for older children with ADHD, AD appears to have a protective function on attention, while for younger children with ADHD, AD seems to worsen their attention problems.

A larger percentage of boys in the samples were related to relatively smaller deficiencies in working memory performance in children with ADHD plus AD as compared to children with ADHD alone. Some previous research suggests that working memory deficits in girls with ADHD are less severe than in boys with ADHD (Seidman et al. 1997). Our meta-analysis seems to suggest that boys with ADHD may benefit more from the protective function of AD on WM.

ADHD guidelines report mixed findings with regard to recommendations in using ADHD medications in children with comorbid ADHD and AD (Physicians Postgraduate Press Inc. 2007). For example, although behavioral intervention and medication alone were similarly effective for children with ADHD and comorbid anxiety, better outcomes were found in children with ADHD and comorbid anxiety who received combined psychosocial treatment and medication (MTA group 1999). On the other hand, adverse effects on anxiety levels and cognitive functions were found for stimulants in children with comorbid ADHD and AD as opposed to non-comorbid group of children with ADHD (Bedard and Tannock 2008; Pliszka 1989; Tannock et al. 1995). Our results suggest better inhibition functioning in medication-naïve children with comorbid ADHD and AD, so an interesting question is whether cognitive functions in children with ADHD and comorbid anxiety are less affected by anxiety in children with ADHD who are ADHD-medication-naïve as opposed to children with ADHD who are familiar with medication. Experimental research is needed selecting groups of children with ADHD with and without comorbid $\mathrm{AD}$, whose performance on executive function tasks is assessed with and without use of ADHD medication in order to shed more light on this possible detrimental effect of ADHD medication on executive functioning in children with ADHD and comorbid AD. An alternative way to shed light on this phenomenon is to experimentally heighten anxiety state levels in children with ADHD with and without ADHD medication when measuring their executive performance.

No other moderating effects were found and comorbid DBD appeared not to be a moderator of the association between attention, response inhibition, and working memory performance tasks and diagnosis group. Different explanations are possible for these results which are mainly related to methodological aspects of the studies included. First, the composition of the samples in different studies was not varied in particular for some moderators. As for the comorbid $\mathrm{DBD}$, in general, comorbid DBD was either present or the information was not available. A second methodological explanation is the fact that for a moderation effect (an interaction) to be found larger sample sizes are required (Hayes 2013). It can further be argued that for an interaction to 
occur, the symptoms (e.g., working memory problems and ODD) should be present in one and the same person, which in case of a heterogeneous disorder such as ADHD is quite challenging. Third, there is also heterogeneity in the tasks and parameters used in our study to assess executive functions; the most tasks were cognitive experimental assessment tools, but also a few neuropsychological (i.e., WISC) tests were used to assess executive functioning.

Finally, inspecting the scarce literature investigating executive functioning in youth with AD may potentially help understand our results. Performance of anxiety-disordered or anxious youth on attention measures such as the one used in the studies in this meta-analysis (i.e., Continuous Performance Test) seems not to be studied previously. Studies investigating attention problems in youth anxiety are concerned with attentional bias paradigm (i.e., shifting attention toward threatening stimuli) (e.g., Puliafico and Kendall 2006). Response inhibition (as studied by the tasks used in this meta-analysis; e.g., Stop Task) has been studied several times. One meta-analytic study (Oosterlaan et al. 1998) found no evidence for enhanced levels of response inhibition in children with AD. In one study included in this meta-analysis (Korenblum et al. 2007), children with $\mathrm{AD}$ were found to have more response inhibition deficits than normal controls, but this association disappeared once comorbid ADHD was taken into account. So previous scarce studies did not found expected enhanced levels of response inhibition in children with $\mathrm{AD}$, and the question is to what extent is inhibition as assessed with laboratory tasks a pure artifact of $\mathrm{AD}$, or a facet that comes to expression in interaction with other disorders such as ADHD. With regard to working memory deficits in anxiety, a recent meta-analytic and theoretical review including child and adult populations found that self-reported and experimentally induced anxiety is associated with poorer performance on working memory measures (Moran 2016). In our meta-analysis we did not find that the ADHD plus AD group had worse performance on measures of working memory as compared to the ADHD alone group, indicating that $\mathrm{AD}$ appears to have a protective function as well in those with ADHD.

\section{Clinical Implications}

Treatment-wise, recent studies have noticed the negative role comorbid ADHD can have in the cognitive behavioral treatment (CBT) of youth with AD (e.g., Halldorsdottir and Ollendick 2014). Comorbid ADHD interferes negatively with mechanisms responsible for CBT success, such as attending to anxiety-provoking stimuli in case of exposure, potentially because of distractibility problems. In line, Maric et al. (2015) found family CBT to be more effective in the long term than child only CBT for children with AD with higher levels of ADHD symptoms. Another study involving children with comorbid ADHD and AD showed positive effects of a combined treatment, that is, parent training plus family-based CBT, on long-term anxiety and ADHD symptoms (Jarrett and Ollendick 2012). So involving parents more in during-treatment activities with this group may be a solution. At the same time, we did not find clear evidence that anxiety worsens EF in children with ADHD; the question remains about the direction of the influence between EF, $\mathrm{AD}$, and $\mathrm{ADHD}$ symptoms. It is perhaps possible that the direction of the influence between ADHD, AD, and EFs is different, in a way that ADHD influences cognitive functioning (attention and working memory) of children with $\mathrm{AD}$ (i.e., increasing attentional biases to threat), and not contrariwise, that $\mathrm{AD}$ influences cognitive functioning of children with ADHD. Consequently, comorbid ADHD should also be a treatment target in the treatment of youth anxiety. Regarding ADHD treatment for primary ADHD problems, an interesting question is whether anxiety should also standard be a treatment target, since our results suggest anxiety to be a protective factor for inhibition problems in children with ADHD, and especially for children who are medication naïve. Our "hunch" would be that clinical levels of anxiety should always be treated as they could be debilitating in all children and in children with ADHD, but that some levels of anxiety are perhaps even desirable in children with ADHD as they could enhance their inhibition, especially if these children were not treated with ADHD medication before. It should be noted that we have found this protective role of anxiety in relation to performance on laboratory tasks in a single session. Future studies should investigate whether this finding also holds for anxiety being protective in everyday life in relation to behavioral manifestations (e.g., impulsivity) of ADHD in children. As appropriate levels of anxiety may probably be an important asset for youth with ADHD, treatment programs for children with comorbid ADHD and AD should pay special attention to education of youth about the possible function of feeling anxious.

Regarding diagnosis, clinicians should assess children for the presence of anxiety when diagnosing ADHD and vice versa. In both cases, attention should be given to executive functioning problems. An interesting question is whether diagnostic assessments should be completed while children are temporarily set off the medication.

\section{Limitations and Future Recommendations}

Whatever the explanation of our results is, it is obvious that more studies are needed in this area using the best assessment tools to assess executive functioning in children with ADHD, ADHD plus AD, AD only, and a control group from the general population. Further, research examining experimental and longitudinal relations between changes in 
executive functions, ADHD symptoms and anxiety symptoms would help shed light on the temporal associations between these variables and provide directions for the most potent treatment targets in children with comorbid ADHD and AD. Finally, it has long been thought that anxiety may mask hyperactivity symptoms in ADHD resulting in ADHD —inattentive subtype — of the disorder (Quay, 1988, 1997). However, recent studies (e.g., MTA group, 1999), involving those included in this meta-analysis, describe presence of anxiety (disorders) in children and adolescents with ADHD-combined subtype, challenging this initial belief. In this meta-analysis, we were not able to investigate the type of ADHD as a moderator of the relation between the diagnosis group and executive functioning, because ADHD - inattentive type - was not specified in the studies included. Thus, this remains as a potentially interesting question for future research areas.

The major limitation of this meta-analysis, that preclude us from drawing firm conclusions, is the limited sample of studies we were able to include, for example, for response inhibition $n=6$. However, the analyses revealed that, although this sample size was relatively small, the sampling variance was small enough to come to the reliable estimate of the mean effect size (CI $-0.74,-0.08)$; so we found evidence for impact of anxiety on inhibition although the six studies show different effect sizes (PI 1.15, 0.33). Further, there was variability among the measures and parameters used to assess attention, response inhibition and working memory functioning, and some variability in the way $\mathrm{AD}$ were defined across studies (i.e., parent interview with and without child involvement, different diagnostic interviews, interviews based on DSM-III, -IV, and 5). As such, this study should be seen and used as hypothesis generating, mainly providing directions for future research endeavors.

Acknowledgements The authors wish to thank Ms. B. Nass who assisted with the literature review, and Mr. J. A. Agelink van Rentergem who provided valuable data-analysis advice.

\section{Compliance with Ethical Standards}

Conflict of interest Authors Marija Maric, Anika Bexkens and Susan M. Bögels declares that they have no conflict of interest.

Ethical Approval This article does not contain any studies with human participants or animals performed by any of the authors.

Open Access This article is distributed under the terms of the Creative Commons Attribution 4.0 International License (http://creativeco mmons.org/licenses/by/4.0/), which permits unrestricted use, distribution, and reproduction in any medium, provided you give appropriate credit to the original author(s) and the source, provide a link to the Creative Commons license, and indicate if changes were made.

\section{References}

\section{Studies marked by an asterisk were included in the meta-regression analysis}

American Psychiatric Association. (2000). Diagnostic and statistical manual of mental disorders, 4th Revised Edition (DSM-IV-TR). Washington, DC: American Psychiatric Association.

American Psychiatric Association. (2013). Diagnostic and statistical manual of mental disorders (5th ed.). Arlington, VA: American Psychiatric Publishing.

Angold, A., Costello, E. J., \& Erkanli, A. (1999). Co-morbidity. Journal of Child Psychology and Psychiatry and Allied Disciplines, $40,57-87$.

Barkley, R. A. (2012). Executive functions: What they are, how they work, and why they evolved. New York: Guilford Press.

Bedard, A.-C., \& Tannock, R. (2008). Anxiety, methylphenidate response, and working memory in children with ADHD. Journal of Attention Disorders, 11, 546-557.

Bögels, S. M. et al. (in preparation). Effects of a mindfulness program for children and adolescents with ADHD and parallel mindful parenting for their parents (MYmind).

Biederman, J., Faraone, S. V., Spencer, T., et al. (1994). Gender differences in a sample of adults with attention deficit hyperactivity disorder. Psychiatry Research, 53, 13-29.

Burgess, G. C., Depue, B. E., Ruzic, L., Willcutt, E. G., Du, Y. P., \& Banich, M. T. (2010). Attentional control activation relates to working memory in attention-deficit/hyperactivity disorder. Biological Psychiatry, 67, 632-640.

Cohen, J. (1992). A power primer. Psychological Bulletin, 112, $155-159$.

Crosbie, J., Arnold, P., Paterson, A., Swanson, J., Dupuis, A., Li, X., et al. (2013). Response inhibition and ADHD traits: Correlates and heritability in a community sample. Journal of Abnormal Child Psychology, 41, 497-507.

Cumyn, L., French, L., \& Hechtman, L. (2009). Comorbidity in adults with attention-deficit hyperactivity disorder. Canadian Journal of Psychiatry, 54, 673-683.

Diamond, I. R., Tannock, R., \& Schachar, R. J. (1999). Response to methylphenidate in children with ADHD and comorbid anxiety. Journal of the American Academy of Child and Adolescent Psychiatry, 38, 402-409.

Egger, M., Smith, D. G., Schneider, M., \& Minder, C. (1997). Bias in meta-analysis detected by a simple, graphical test. British Medical Journal, 315, 629-634.

Field, A. (2009). Discovering statistics using SPSS. London: Sage Publications Ltd.

Field, A. P., \& Gillett, R. (2010). How to do a meta-analysis. British Journal of Mathematical and Statistical Psychology, 63, 665-694.

Greenhill, L., Kollins, S., Abikoff, H., McCracken, J., Riddle, M., Swanson, J., et al. (2006). Efficacy and safety of immediaterelease methylphenidate treatment for preschoolers with ADHD. Journal of the American Academy Child and Adolescent Psychiatry, 45, 1284-1293.

Habeck, C. W., \& Schultz, A. K. (2015). Community-level impacts of white-tailed deer on understorey plants in North American forests: A meta-analysis. AoB Plants, 7, plv119.

Hayes, A. F. (2013). Introduction to mediation, moderation, and conditional process analysis: A regression-based approach. New York: The Guilford Press.

IntHout, J., Ioannidis, J. P., Rovers, M. M., \& Goeman, J. J. (2016). Plea for routinely presenting prediction intervals in meta-analysis. British Medical Journal Open, 6, e010247. 
Jarrett, M. A., \& Ollendick, T. H. (2008). A conceptual review of the comorbidity of attention-deficit/hyperactivity disorder and anxiety: Implications for future research and practice. Clinical Psychology Review, 28, 1266-1280.

Jarrett, M. A., \& Ollendick, T. H. (2012). Treatment of comorbid attention-deficit/hyperactivity disorder and anxiety in children: A multiple baseline design analysis. Journal of Consulting and Clinical Psychology, 80, 239-244.

*Jarrett, M. A., Wolff, J. C., Davis, T. E., Cowart, M. J., \& Ollendick, T. H. (2016). Characteristics of children with ADHD and comorbid anxiety. Journal of Attention Disorders, 20, 636-644.

Kessler, R. C., Adler, L., Barkley, R., Biederman, J., Conners, C. K., Demler, O., et al. (2006). The prevalence and correlates of adult ADHD in the United States: Results from the National Comorbidity Survey Replication. American Journal of Psychiatry, 163, 716-723.

Kofler, M. J., Rapport, M. D., Bolden, J., Sarver, D. E., \& Raiker, J. S. (2010). ADHD and working memory: The impact of central executive deficits and exceeding storage/rehearsal capacity on observed inattentive behavior. Journal of Abnormal Child Psychology, 38, 149-161.

*Korenblum, C. B., Chen, S. X., Manassis, K., \& Schachar, R. J. (2007). Performance monitoring and response inhibition in anxiety disorders with and without comorbid ADHD. Depression and Anxiety, 24, 227-232.

*Manassis, K., Tannock, R., \& Barbosa, J. (2000). Dichotic listening and response inhibition in children with comorbid anxiety disorders and ADHD. Journal of the American Academy of Child and Adolescent Psychiatry, 39, 1152-1159.

*Manassis, K., Tannock, R., Young, A., \& Francis-John, S. (2007). Cognition in anxious children with attention deficit hyperactivity disorder: A comparison with clinical and normal children. Behavioral and Brain Functions, 3, 4.

Mancini, C., Van Ameringen, M., Oakman, J. M., \& Figueiredo, D. (1999). Childhood attention deficit/hyperactivity disorder in adults with anxiety disorders. Psychological Medicine, 29, 515-525.

Maric, M., van Steensel, F. J. A., \& Bögels, S. M. (2015). Parental involvement in CBT for anxiety-disordered youth revisited: Family CBT outperforms child CBT in the long term for children with comorbid ADHD symptoms. Journal of Attention Disorders, 1-9.

Moher, D., Liberati, A., Tetzlaff, J., \& Altman, D. G. (2009). Preferred reporting items for systematic reviews and meta-analyses: The PRISMA statement. Journal of Clinical Epidemiology, 62, $1006-1012$.

Moran, T. P. (2016). Anxiety and working memory capacity: A meta-analysis and narrative review. Psychological Bulletin, 142, $831-864$.

MTA Cooperative Group. (1999). Moderators and mediators of treatment response for children with attention-deficit/hyperactivity disorder. Archives of General Psychiatry, 56, 1088-1096.

Mueller, A. K., Fuermaier, A. B., Koerts, J., \& Tucha, L. (2012). Stigma in attention deficit hyperactivity disorder. Attention Deficit and Hyperactivity Disorders, 4, 101-114.

*Newcorn, J. H., et al. (2001). Symptom profiles in children with ADHD: Effects of comorbidity and gender. Journal of the American Academy Child and Adolescent Psychiatry, 40, 137-146.

Nigg, J. T. (2006). What causes ADHD? Understanding what goes wrong and why. New York: Guilford Press.

Ollendick, T. H., Jarrett, M. A., Grills-Taquechel, A. E., Hovey, L. D., $\&$ Wolff, J. C. (2008). Comorbidity as a predictor and moderator of treatment outcome in youth with anxiety, affective, attention deficit/hyperactivity disorder, and oppositional/conduct disorders. Clinical Psychology Review, 28, 1447-1471.

Oosterlaan, J., Logan, G. D., \& Sergeant, J. A. (1998). Response Inhibition in $\mathrm{AD} / \mathrm{HD}, \mathrm{CD}$, comorbid $\mathrm{AD} / \mathrm{HD}+\mathrm{CD}$, anxious, and control children: A meta-analysis of studies with the stop task.
The Journal of Child Psychology and Psychiatry and Allied Disciplines, 39, 411-425.

Park, S., Cho, M. J., Chang, S. M., Jeon, H. J., Ch, S.-J., Kim, B.-S., et al. (2011). Prevalence, correlates, and comorbidities of adult ADHD symptoms in Korea: Results of the Korean epidemiologic catchment area study. Psychiatry Research, 186, 378-383.

Partlett, C., \& Riley, R. D. (2017). Random effects meta-analysis: Coverage performance of $95 \%$ confidence and prediction intervals following REML estimation. Statistics in Medicine, 36, 301-317.

Physicians Postgraduate Press Inc. (2007). Managing ADHD in children, adolescents, and adults with comorbid anxiety in primary care. The Primary Care Companion to The Journal of Clinical Psychiatry, 9, 129-138.

*Pliszka, S. R. (1992). Comorbidity of attention-deficit hyperactivity disorder and overanxious disorder. Journal of the American Academy Child and Adolescent Psychiatry, 31, 197-203.

Pliszka, S. R. (1989). Effect of anxiety on cognition, behavior, and stimulant response in ADHD. Journal of American Academy Child and Adolescent Psychiatry, 28, 882-887.

Pliszka, S. R., Carlson, C., \& Swanson, J. M. (1999). ADHD with comorbid disorders: Clinical assessment and management. New York: Guilford.

Posner, K., Melvin, G. A., Murray, D. W., et al. (2007). Clinical presentation of ADHD in preschool children: Preschoolers with ADHD treatment study (PATS). Journal of Child and Adolescent Psychopharmacology, 17, 547-562.

Puliafico, A. C., \& Kendall, P. C. (2006). Threat-related attentional bias in anxious youth: A review. Clinical Child and Family Psychology Review, 9, 162-180.

Quay, H. C. (1988). The behavioral reward and inhibition system in childhood behavior disorder. In J. A. Bloomingdale (Ed.), Attention deficit disorder (Vol. 3, p. 12). Oxford: Pergamon Press.

Quay, H. C. (1997). Inhibition and attention deficit hyperactivity disorder. Journal of Abnormal Child Psychology, 25, 7-13.

*Rodríguez, C., González-Castro, P., García, T., Núñez, J. C., \& Alvarez, L. (2014). Attentional functions and trait anxiety in children with ADHD. Learning and Individual Differences, 35, 147-152.

Rucklidge, J. J., \& Tannock, R. (2001). Psychiatric, psychosocial, and cognitive functioning of female adolescents with ADHD. Journal of the American Academy Child and Adolescent Psychiatry, 40, 530-540.

Sarver, D. E., Rapport, M. D., Kofler, M. J., Raiker, J. S., \& Friedman, L. M. (2015). Hyperactivity in attention-deficit/hyperactivity disorder (ADHD): Impairing deficit or compensatory behavior? Journal of Abnormal Child Psychology, 43, 1219-1232.

Schatz, D. B., \& Rostain, A. L. (2006). ADHD with comorbid anxiety: A review of the current literature. Journal of Attention Disorders, $10,141-149$.

Schoemaker, K., Mulder, H., Dekovic, M., \& Matthys, W. (2013). Executive functions in preschool children with externalizing behavior problems: A meta-analysis. Journal of Abnormal Child Psychology, 41, 457-471.

Seidman, L. J., Biederman, J., Faraone, S. V., \& Weber, W. (1997). A pilot study of neuropsychological function in girls with ADHD. Journal of the American Academy of Child Psychiatry, 36, 366-373.

Seidman, L. J., Biederman, J., Monuteaux, M. C., Valera, E., Doyle, A. E., \& Faraone, S. V. (2005). Impact of sex and age on executive functioning: Do girls and boys with and without attention deficit hyperactivity disorder differ neuropsychologically in preteen and teenage years? Developmental Neuropsychology, 27, 79-105.

Smalley, S. L., McGough, J. J., Moilanen, I. K., Loo, S. K., Taanila, A., Ebeling, H., et al. (2007). Prevalence and psychiatric comorbidity of attention-deficit/hyperactivity disorder in an adolescent Finnish population. Journal of the American Academy Child and Adolescent Psychiatry, 46, 1575-1583. 
Sobanski, E., Brüggemann, D., Alm, B., Kern, S., Deschner, M., Schubert, T., et al. (2007). Psychiatric comorbidity and functional impairment in a clinically referred sample of adults with attention-deficit/hyperactivity disorder (ADHD). European Archives of Psychiatry and Clinical Neuroscience, 257, 371-377.

Sørensen, L., Plessen, K. J., Nicholas, J., \& Lundervold, A. J. (2011). Is behavioral regulation in children with ADHD aggravated by comorbid anxiety disorder? Journal of Attention Disorders, 15, 56-66.

Tannock, R. (2009). ADHD with anxiety disorders. In T. E. Brown (Ed.), ADHD comorbidities: Handbook for ADHD complications in children and adults (pp. 131-155). Arlington, VA: American Psychiatric Publishing.

Tannock, R., Ickowicz, A., \& Schachar, R. (1995). Differential effects of methylphenidate on working memory in ADHD children with and without comorbid anxiety. Journal of the American Academy Child and Adolescent Psychiatry, 34, 886-896.

Tillman, C. M., Eninger, L., Forssman, L., \& Bohlin, G. (2011). The relation between working memory components and ADHD symptoms from a developmental perspective. Developmental Neuropsychology, 36, 181-198.
*Trani, M., Casini, M. P., Capuzzo, F., Gentile, S., Bianco, G., Menghini, D., et al. (2011). Executive and intellectual functions in attention-deficit/hyperactivity disorder with and without comorbidity. Brain and Development, 33, 462-469.

*Vance, A., Ferrin, M., Winther, J., \& Gomez, R. (2013). Examination of spatial working memory performance in children and adolescents with attention deficit hyperactivity disorder, combined type (ADHD-CT) and anxiety. Journal of Abnormal Child Psychology, 41, 891-900.

Viechtbauer, W. (2010). Conducting meta-analyses in R with the metafor package. Journal of Statistical Software, 36, 1-48.

*Vloet, T. D., Konrad, K., Herpertz-Dahlmann, B., Polier, G. G., \& Günther, T. (2010). Impact of anxiety disorders on attentional functions in children with ADHD. Journal of Affective Disorders, 124, 283-290.

*Yurtbasi, P., Aldemir, S., Teksin Bakir, M. G., Aktas, S., Ayvaz, F. B., Pistav Satilmis, S., \& Münir, K. (2015). Comparison of neurological and cognitive deficits in children with ADHD and anxiety disorders. Journal of Attention Disorders, 1-14. 